\title{
Analyticity of the time dependence of resonance poles: Solving the Friedrichs model with a time-dependent interaction
}

\author{
O. Civitarese ${ }^{1}$ and M. Gadella ${ }^{2}$ \\ ${ }^{1}$ Department of Physics, University of La Plata, c.c. 67 (1900) La Plata, Argentina \\ ${ }^{2}$ Departament of FTAO, Facultad de Ciencias, University of Valladolid, 46071 Valladolid, Spain
}

(Received 10 May 2012; published 8 August 2012)

\begin{abstract}
We extend the standard Friedrichs model with an extra term that includes time-dependent interactions. The time dependence of the poles of the reduced resolvent of the model is explicitly calculated. It is found that these poles behave as analytical functions of the added time-dependent interaction. The present results are compared with the ones reported by Kälbermann, concerning the assisted tunnelling of $\alpha$ particles.
\end{abstract}

DOI: 10.1103/PhysRevC.86.024602

PACS number(s): 24.30.-v, 73.40.Gk, 25.70.Ef

\section{INTRODUCTION}

The study of the physical and mathematical aspects of resonance poles has been the subject of continuous effort since the physical consequences of their existence were dramatically stressed by Gamow in the earlier days of quantum mechanics [1]. In the modern literature, the role of resonance poles in scattering of particles by nuclei and in the decay of nuclei has been analyzed intensively [2-4]. The connection between mathematical [5] and physical formulations of the problem was investigated in Ref. [6] and further explored in a series of papers by several authors [7-11]. Relevant applications of the concept of resonance poles in nuclear structure and nuclear reactions can be found in Refs. $[3,12,13]$.

Generally speaking, the concept of resonance poles is tied up to the $S$-matrix formalism [5,14,15]. The Hamiltonian formulation is, perhaps, better presented in the model of Friedrichs [16]. For a detailed discussion of this model and its solutions the reader is kindly referred to a recent review article [17].

Further generalizations of the Friedrichs model, with applications to nuclear physics, have been introduced in Refs. [18,19].

In this work we shall address, from a mathematical oriented view, the question of the enhancement (or hindrance) of the resonant structure of a state, as suggested by Kälbermann [20,21]. In these papers, Kälbermann has discussed, based on numerical analysis, the assisted tunneling of a wave packet between square barriers, and concluded that the tunneling probability is enhanced by the perturbation [20,21].

In order to verify this finding, we shall frame the questions raised in Refs. [20,21] in the language of the Friedrichs model. We shall add, to the standard Friedrichs model, a time-dependent interaction, and feature the solutions in terms of the parameters of such an interaction. The aim of the paper is, therefore, to probe the conditions under which a resonance can be modified by the interaction with external potentials (or fields), without depending much on the detail of the interactions.

The present paper is organized as follows. In Sec. II we review, for the benefit of the readers, the elements entering the standard Friedrichs model. Section III is devoted to the mathematical formulation of a one-dimensional model, corresponding to a finite square well to which we have added a delta-force-type interaction. Although this is a particular and very specific model, it shows that the enhancement or reduction of the lifetime depends solely on certain parameters of the added interaction. Section IV describes the extension of the Friedrichs model, which we have developed to accommodate a time-dependent interaction. Finally, our conclusions are drawn in Sec. V.

\section{FORMALISM}

The basic Friedrichs model is the simplest nontrivial exactly solvable test model for resonances [16]. It admits rather simple generalizations that may be used as excellent tools to test a resonance behavior with a wide sort of interactions. For instance, we have used it in the past in order to study couplings of fermions with bosons and boson fields [6,19]. A presentation of some of the most relevant generalizations of this model is given in Ref. [17].

A brief description of the Friedrichs model is presented here for the sake of completeness. This description is given in the energy representation, so that dimensional problems are avoided. As in any process producing resonances one has two Hamiltonians: a free or unperturbed Hamiltonian $H_{0}$ and a total or perturbed Hamiltonian $H=H_{0}+\lambda V$, where $V$ is the potential describing the interaction and $\lambda$ is a coupling constant, which is usually chosen positive. $H_{0}$ has a simple continuous spectrum given by $\mathbb{R}^{+}=[0, \infty)$ and a bound state with energy $\omega_{0}>0$. The potential $V$ intertwines the discrete and continuous spectrum of $H_{0}$. This interaction can be regarded as an interaction between a discrete boson and a boson field. Thus, in the energy representation, we can write

$$
\begin{aligned}
H_{0} & =\omega_{0}|1\rangle\left\langle 1\left|+\int_{0}^{\infty} \omega\right| \omega\right\rangle\langle\omega| d \omega, \\
V & =\int_{0}^{\infty} f(\omega)[|\omega\rangle\langle 1|+| 1\rangle\langle\omega|] d \omega .
\end{aligned}
$$

Here, $H_{0}|1\rangle=\omega_{0}|1\rangle, H_{0}|\omega\rangle=\omega|\omega\rangle$, with $\omega \in[0, \infty)$, and $f(\omega)$ is a given function, hereafter referred to as the "form factor" of the interaction $V$. 
Note that the eigenvalue of $H_{0}$ is embedded in its continuous spectrum. As a consequence of the interaction, the bound state is dissolved in the continuum and a resonance is produced. In order to obtain the resonances, we consider the restricted resolvent

$$
\frac{1}{\eta(z)}=\left\langle 1\left|\frac{1}{H-z}\right| 1\right\rangle, \quad \eta(z)=z-\omega_{0}-\lambda^{2} \int_{0}^{\infty} \frac{|f(\omega)|^{2}}{z-\omega} d \omega .
$$

The function $\eta(z)$ is analytic and it admits a branch cut coinciding with the positive semiaxis $[0, \infty)$ and can be analytically continued through the cut, both from above to below and from below to above. Resonances are precisely the zeros of these analytic continuations, which appear in complex conjugate pairs. Each pair determines one and only one resonance. In the basic Friedrichs model that we are describing here, there is only one pair of resonant zeros usually of simple multiplicity. The boundary values on the positive semiaxis are respectively given by

$$
\eta_{ \pm}(x)=x-\omega_{0}-\lambda^{2} \int_{0}^{\infty} \frac{|f(\omega)|^{2}}{x-\omega \pm i 0} d \omega .
$$

The interacting Hamiltonian $H=H_{0}+\lambda V$ possesses a simple continuous spectrum coinciding with the positive semiaxis $\mathbb{R}^{+}=[0, \infty)$. Corresponding to the "in" and "out" states of scattering theory, there is a pair of complete sets of eigenvectors of $H$, usually denoted as $\left|\omega^{-}\right\rangle$and $\left|\omega^{+}\right\rangle$, so that

$$
H=\int_{0}^{\infty} \omega\left|\omega^{ \pm}\right\rangle\left\langle\omega^{ \pm}\right| d \omega
$$

where $H\left|\omega^{ \pm}\right\rangle=\omega\left|\omega^{ \pm}\right\rangle$. Vectors $\left|\omega^{ \pm}\right\rangle$are called the in and the out eigenkets of $H$. They can be written in terms of the eigenvectors $|1\rangle$ and $|\omega\rangle$ of the unperturbed Hamiltonian $H_{0}$ as [7]

$$
\left|\omega^{ \pm}\right\rangle=|\omega\rangle+\frac{\lambda f(\omega)}{\eta_{ \pm}(\omega)}\left(|1\rangle+\int_{0}^{\infty} d \omega^{\prime} \frac{\lambda f\left(\omega^{\prime}\right)}{\omega-\omega^{\prime} \pm i 0}\left|\omega^{\prime}\right\rangle\right) .
$$

The Friedrichs model admits a well defined $S$ matrix [22], and the poles of the analytic continuation of the $S$ matrix through the positive semiaxis coincide with the zeros of the continuation of $\eta(z)$. Thus, poles of the $S$ matrix and poles of the reduced resolvent coincide for the Friedrichs model. Both types of poles are taken as usual definitions for resonance poles. They are located at the conjugate complex numbers $z_{R}=E_{D}-i \Gamma / 2$ and $z_{R}^{*}=E_{D}+i \Gamma / 2$, where $E_{D}$ is the resonant energy and $\Gamma$ the width. The Gamow vectors $\psi^{D}$ and $\psi^{G}$ are the eigenvectors (in a generalized sense, see Ref. [6]) of $H$ with eigenvalues $z_{R}$ and $z_{R}^{*}$, i.e., $H \psi^{D}=z_{R} \psi^{D}$ and $H \psi^{G}=z_{R}^{*} \psi^{G}$, respectively. The explicit form for these vectors in the Friedrichs model is given by the expressions

$$
\begin{aligned}
\psi^{D} & =|1\rangle+\int_{0}^{\infty} \frac{\lambda f(\omega) d \omega}{z_{R}-\omega+i 0}|\omega\rangle d \omega, \\
\psi^{G} & =|1\rangle+\int_{0}^{\infty} \frac{\lambda f(\omega) d \omega}{z_{R}^{*}-\omega-i 0}|\omega\rangle d \omega .
\end{aligned}
$$

Both $\psi^{D}$ and $\psi^{G}$ are usually taken as the vector state for the resonance. Note that neither $\psi^{D}$ nor $\psi^{G}$ can be normalized in the usual sense. The meaning of the $\pm i 0$ factor which appears in the denominators of Eqs. (6) and (7) is standard in the theory of distributions.

\section{A FINITE SQUARE-WELL POTENTIAL PLUS A DIRAC DELTA}

Let us consider a finite one-dimensional square well with a hard core at the origin plus a Dirac delta located beyond the well. The Hamiltonian of the system has the following form:

$$
H=-\frac{\hbar^{2}}{2 m} \frac{d^{2}}{d x^{2}}+V(x)+\gamma \delta(x-c),
$$

with

$$
V(x)= \begin{cases}0 & \text { if } 0<x<a, \\ U_{0}>0 & \text { if } a \leqslant x \leqslant b, \\ 0 & \text { if } b>0\end{cases}
$$

and $c>b$.

Our goal is to compare the width of the lowest energy resonance without the presence of the perturbation in (8), in this case represented by the delta interaction, to the same results when we have included the delta interaction. Note that, in principle, the contribution of the delta could be either repulsive $\gamma>0$ or attractive $\gamma<0$. In the latter case, it appears another resonance due to the delta that we are not going to consider. The situation without the delta has been studied for instance in [23]. Here, we made a similar analysis including the last term in (8).

Then, the positive semiaxis can be divided into four intervals $[0, a],[a, b],[b, c]$ and $[c, \infty]$, that we denote as I, II, III, and IV intervals, respectively, for which the wave function has the following form:

$$
\begin{aligned}
\psi_{I}(x) & =\tilde{A} \sin (k x), \quad 0<x<a, \\
\psi_{I I}(x) & =\tilde{B} e^{-Q x}+\tilde{C} e^{Q x}, \quad a<x<b, \\
\psi_{I I I}(x) & =\tilde{D} e^{i k c}+\tilde{E} e^{-i k c}, \quad b<x<c, \\
\psi_{I V}(x) & =\tilde{F} e^{i k x}, \quad x>c .
\end{aligned}
$$

Here,

$$
k=\sqrt{\frac{2 m}{\hbar^{2}} E}, \quad Q=\sqrt{\frac{2 m}{\hbar^{2}}\left(U_{0}-E\right)}, \quad E=E_{D}-i \frac{\Gamma}{2},
$$

where $E_{D}$ represents the resonant energy and $\Gamma$ is the width.

For the determination of the resonances, we applied the so called purely outgoing boundary conditions according to which for the values of $k$ for which we find a resonance there is no incoming wave.

At the points $a$ and $b$ we apply the standard matching conditions, i.e., continuity of the wave function and its derivative. At the point $c$ we impose the continuity of the 
wave function and

$$
\begin{aligned}
& \psi_{I V}^{\prime}(c)-\psi_{I I I}^{\prime}(c) \\
& \quad=\gamma \psi_{I V}(c) \Longleftrightarrow i k \tilde{F} e^{i k c}-i k \tilde{D} e^{i k c}+i k \tilde{E} e^{-i k c} \\
& \quad=\gamma \tilde{F} e^{i k c} .
\end{aligned}
$$

Then, we write $\tilde{E}$ and $\tilde{D}$ in terms of $\tilde{A}$ and $\tilde{F}$. After some cumbersome although straightforward calculation, this gives two expressions of $\tilde{F}$ in terms of $\tilde{A}$ :

$$
\begin{aligned}
\tilde{F}= & -\frac{e^{-i k b}}{2 i k-\gamma} \frac{1}{2 Q}\left\{e^{-Q(b-a)}(Q-i k)[Q \sin (k a)\right. \\
& -k \cos (k a)]-e^{Q(b-a)}(Q+i k)[Q \sin k a \\
& +k \cos (k a)]\} \tilde{A} \\
\tilde{F}= & \frac{e^{i k b} e^{-2 i k c}}{\gamma} \frac{1}{2 Q}\left\{e^{-Q(b-a)}(Q+i k)[Q \sin (k a)\right. \\
& -k \cos (k a)]-e^{Q(b-a)}(Q-i k)[Q \sin (k a) \\
& +k \cos (k a)]\} \tilde{A}
\end{aligned}
$$

Both expressions must be identical, which after some manipulations gives

$$
\begin{aligned}
& {\left[\frac{Q}{k} \tan (k a)+1\right]\left[\frac{e^{-2 i k b} e^{-2 i k c} \gamma}{\gamma-2 i k} \frac{Q+i k}{Q-i k}-1\right]} \\
& \quad=e^{-2 Q(b-a)}\left[\frac{Q}{k} \tan (k a)-1\right]\left[\frac{e^{-2 i k b} e^{-2 i k c} \gamma}{\gamma-2 i k}-\frac{Q+i k}{Q-i k}\right] .
\end{aligned}
$$

A similar analysis was made in Refs. [23] and [24]. In both references the authors have considered the Hamiltonian given in Eq. (8) with; that is, without the inclusion of a Diracdelta term. Then, the resulting consistency equation is written $[23,24]$

$$
\left[\frac{Q}{k} \tan (k a)+1\right]=e^{-2 Q(b-a)}\left[\frac{Q}{k} \tan (k a)-1\right]\left[\frac{Q+i k}{Q-i k}\right] .
$$

which is precisely the value of Eq. (15) in the limit $\gamma=0$.

Then, we define

$$
k_{0}:=\sqrt{\frac{2 m}{\hbar^{2}} E_{D}}, \quad Q_{0}:=\sqrt{\frac{2 m}{\hbar^{2}}\left(U_{0}-E_{D}\right)}
$$

and write the following expressions in the first order of $\Gamma$ :

$$
\begin{aligned}
e^{-2 i k(b+c)} & =1-2 i(b+c)\left(k_{0}-\frac{i m \Gamma}{2 \hbar^{2} k_{0}}\right) \\
\frac{1}{\gamma-2 i k} & =\frac{1}{\gamma}\left[\frac{1}{1-\frac{2 i k}{\gamma}}\right]=\frac{1}{\gamma}\left[1+\frac{2 i}{\gamma}\left(k_{0}-\frac{i m \Gamma}{2 \hbar^{2} k_{0}}\right)\right],
\end{aligned}
$$

and

$$
\frac{Q+i k}{Q-i k}=\frac{(Q+i k)^{2}}{Q^{2}+k^{2}} \simeq \frac{\left(Q_{0}+i k_{0}\right)^{2}}{Q_{0}^{2}+k_{0}^{2}} .
$$

In order to make our analysis computationally simpler we make the following approximations, which are reasonable due to the specific form of the potential well: i) $U_{0} \gg E_{D}$, so that the resonant energy is much smaller than the barrier height, this directly implies that $Q_{0} \gg k_{0}$ and that $Q_{0}^{2}+k_{0}^{2} \simeq Q_{0}^{2}$, and ii) we assume that the barrier width is large, so that $Q_{0}(b-$ a) $\gg 0$. As a consequence of the latter assumption the system can be considered as quasi stationary [23,24]. From (15), this shows that $1+\left(Q_{0} / k_{0}\right) \tan \left(k_{0} a\right) \simeq 0$. Carrying out all these approximations into (15), we obtain the following expression:

$$
\begin{aligned}
& \left(\frac{Q_{0} a}{k_{0} \cos ^{2}\left(k_{0} a\right)}-\frac{Q_{0} \tan \left(k_{0} a\right)}{k_{0}^{2}}\right)\left(\frac{-m \Gamma}{2 \hbar^{2} k_{0}}\right) \\
& =e^{-2 Q_{0}(b-a)}\left[\frac{Q_{0}}{k_{0}} \tan \left(k_{0} a\right)-1\right]\left[\frac{2 Q_{0} k_{0}}{Q_{0}^{2}+k_{0}^{2}}+\gamma B_{0}\right],
\end{aligned}
$$

with

$$
\begin{aligned}
2 k_{0} Q_{0} B_{0}= & 2 Q_{0} k_{0} \cos \left[2 k_{0}(b+c)\right]-Q_{0} \sin \left[2 k_{0}(b+c)\right] \\
& +Q_{0}^{2} \cos \left[2 k_{0}(b+c)\right]+4 Q_{0}^{2} k_{0}^{2} \sin \left[2 k_{0}(b+c)\right] .
\end{aligned}
$$

Equation (21) differs from the similar result obtained in Refs. [23] and [24] in the term in $\gamma B_{0}$. We observe that this term is oscillating and that it depends on $b$ and $c$, i.e., the end of the barrier and the point supporting the delta interaction.

Equation (21) gives the following approximation for the width of the resonances fulfilling the considered conditions:

$$
\Gamma \simeq \frac{4 k_{0}^{2} \hbar^{2}}{\left(Q_{0} a+1\right) m} e^{-2 Q_{0}(b-a)}\left[\frac{2 k_{0}}{Q_{0}}+\gamma B_{0}\right] .
$$

Note that the quasistationarity gives $k_{0} \simeq(\pi n) / a$, which implies that $Q_{0} a \simeq\left(Q_{0} n \pi\right) / k_{0} \gg 1$, since $n \neq 0$. Then, Eq. (23) takes its final form as

$$
\Gamma \simeq \frac{4 k_{0}^{2} \hbar^{2}}{m Q_{0} a} e^{-2 Q_{0}(b-a)}\left[\frac{2 k_{0}}{Q_{0}}+\gamma B_{0}\right] .
$$

It is straightforward to compare this expression with the expression obtained in Ref. [23] for the same model without the delta-force interaction, which is

$$
\Gamma \simeq \frac{4 k_{0}^{2} \hbar^{2}}{m Q_{0} a} e^{-2 Q_{0}(b-a)}\left[\frac{2 k_{0}}{Q_{0}}\right]
$$

As we can see from Eqs. (22), (24), and (25), the addition of a Dirac delta changes, at the same level of accuracy, the value of the resonance width by a term which solely depends on the location of the values $b$ (end of the barrier) and $c$ (support of the delta) and, of course, the delta amplitude $\gamma$. The additional term could be either positive (width becomes larger) or negative (width becomes smaller). When $\gamma=0$ (no Dirac-delta time-dependent interaction is present in the model Hamiltonian), the results of the standard theory of resonances are recovered [23,24].

\section{A RESONANCE (GAMOW STATE) INTERACTING WITH THE CONTINUUM}

Let us go back to the Friedrichs model introduced earlier. The Friedrichs model gave us vector states for the resonance state. Now, assume that this resonance interacts with the continuum corresponding to the total Hamiltonian $H$ in the same manner that the bound state of the unperturbed 
Hamiltonian $H_{0}$ interacts with its continuous spectrum. The procedure used there gives us the idea on how to proceed further. In fact, we can define a new interaction given by the following potential:

$$
V_{1}(t):=\int_{0}^{\infty} g(\omega, t)\left[\left|\omega^{ \pm}\right\rangle\left\langle\psi^{G}|+| \psi^{D}\right\rangle\left\langle\omega^{ \pm}\right|\right] d \omega .
$$

Here, we have chosen a time-dependent form factor $g(\omega, t)$ due to the necessary comparison to the results in Ref. [20]. Now, the Hamiltonian becomes time dependent and it is given by $W(t):=H_{0}+\lambda V+\gamma V_{1}(t)$, where $\gamma$ is a positive coupling constant.

The coupling constants $\lambda$ and $\gamma$ should be smaller than $\omega_{0}$ because we want to obtain reasonably good results at the first order of approximation in these couplings. In addition, in the standard Friedrichs model the resonance is an analytic function of $\lambda$ given some conditions on the form factor [22]. This, in particular, means for that small $\lambda$ we have a resonant energy $E_{D}$ near to $\omega_{0}$ and a small width $\Gamma$, which implies a longer lifetime.

Our goal is to evaluate the resonances for the Hamiltonian pair $\{H, W(t)\}$. With this objective in mind, we write the following eigenvalue equation:

$$
\left\{H_{0}+\lambda V+\gamma V_{1}(t)-z\right\} \psi(z)=0
$$

where the vector state $\psi(z)$ should have a span in terms of the eigenvectors of $H_{0}$ :

$$
\psi(z)=\beta(z)|1\rangle+\int_{0}^{\infty} \phi(\omega, z)|\omega\rangle d \omega
$$

Then, the searched resonances should be the poles of the function $\beta(z)$.

In order to calculate the functions $\beta(z)$ and $\phi(\omega, z)$, we proceed as follows. First of all we ignore the term $V_{1}(t)$. Then, the remaining terms correspond to the standard Friedrichs model, so that

$$
\begin{aligned}
& \left\{H_{0}+\lambda V-z\right\} \psi(z) \\
& =\left\{\left(\omega_{0}-z\right) \beta(z)+\lambda \int_{0}^{\infty} f(\omega) \phi(\omega, z) d \omega\right\}|1\rangle \\
& \quad+\int_{0}^{\infty}\{(\omega-z) \phi(\omega, z)+\lambda f(\omega) \beta(z)\}|\omega\rangle d \omega .
\end{aligned}
$$

Next, we compute $V_{1}(t) \psi(z)$. To proceed with this calculation, we should take into account that in the standard Friedrichs model the eigenvector $|1\rangle$ is taken to be normalized and that the spaces corresponding to the discrete and continuous spectrum of $H_{0}$ must be mutually orthogonal as $H_{0}$ is self adjoint, and also that the continuous spectrum of $H_{0}$ is simple so that

$$
\langle 1 \mid 1\rangle=1, \quad\langle\omega \mid 1\rangle=0, \quad\left\langle\omega \mid \omega^{\prime}\right\rangle=\delta\left(\omega-\omega^{\prime}\right),
$$

for all $\omega, \omega^{\prime} \in[0, \infty)$ and $\delta(x)$ is the Dirac delta.
Furthermore, we are using a first-order approximation so that we shall get rid of all terms of second and higher orders in the couplings.

Lets us apply $V_{1}(t)$ to $|1\rangle$ first. Using Eq. (3), we have

$$
\begin{aligned}
\left\langle\omega^{ \pm} \mid 1\right\rangle= & \langle\omega \mid 1\rangle+\frac{\lambda f(\omega)}{\eta_{\mp}(\omega)} \\
& \times\left\{\langle 1 \mid 1\rangle+\int_{0}^{\infty} d \omega^{\prime} \frac{\lambda f\left(\omega^{\prime}\right)}{\omega-\omega^{\prime} \mp i 0}\left\langle\omega^{\prime} \mid 1\right\rangle\right\} \\
= & \frac{\lambda f(\omega)}{\eta_{\mp}(\omega)},
\end{aligned}
$$

where obviously from Eq. (3), $\eta_{\mp}(\omega)=\eta_{ \pm}{ }^{*}(\omega)$. Then, we use Eq. (5):

$$
\left\langle\psi^{G} \mid 1\right\rangle=\langle 1 \mid 1\rangle+\int_{0}^{\infty} \frac{\lambda f(\omega) d \omega}{z_{R}-\omega+i 0}\langle\omega \mid 1\rangle=1,
$$

so that

$$
V_{1}(t)|1\rangle=\int_{0}^{\infty} g(\omega, t)\left[\left|\omega^{ \pm}\right\rangle+\frac{\lambda f(\omega)}{\eta_{\mp}(\omega)}\left|\psi^{D}\right\rangle\right] d \omega .
$$

Next for all $\alpha \in[0, \infty)$, we compute

$$
\left\langle\omega^{ \pm} \mid \alpha\right\rangle=\delta(\omega-\alpha)+\lambda^{2} \frac{f(\omega)}{\eta_{\mp}(\omega)} \frac{f(\alpha)}{\omega-\alpha \mp i 0} \simeq \delta(\omega-\alpha) .
$$

Note that we get rid of the terms with order $\lambda^{2}, \gamma^{2}$, or $\lambda \gamma$, which justifies the approximation in Eq. (34). Also,

$$
\left\langle\psi^{G} \mid \alpha\right\rangle=\frac{\lambda f(\alpha)}{z_{R}-\alpha+i 0},
$$

so that

$$
\begin{aligned}
V_{1}(t) \int_{0}^{\infty} \phi(\omega, z)|\omega\rangle d \omega= & \frac{\lambda f(\alpha)}{z_{R}-\alpha+i 0} \int_{0}^{\infty} g(\omega, t)\left|\omega^{ \pm}\right\rangle d \omega \\
& +\left[\int_{0}^{\infty} g(\omega, t) d \omega\right]\left|\psi^{D}\right\rangle
\end{aligned}
$$

The next step is to use the span (5) of $\left|\omega^{ \pm}\right\rangle$and the span (6) of $\left|\psi^{D}\right\rangle$ and then deleting all second-order terms in the coupling constants. Then, we carry all this into Eq. (27) and use Eq. (29). We obtain an expression of the form $A|1\rangle+\int_{0}^{\infty} B|\omega\rangle d \omega=0$. Equating $A=0$ and $B=0$, we have the following pair of equations:

$$
\begin{aligned}
\left(\omega_{0}-z\right) \beta(z)+\int_{0}^{\infty} d \omega \phi(\omega, z)[\gamma g(\omega, t)+\lambda f(\omega)] & =0 \\
(\omega-z) \phi(\omega, z)+\beta(z)[\lambda f(\omega)+\gamma g(\omega, t)] & =0 .
\end{aligned}
$$

From Eq. (38), we obtain

$$
\phi(\omega, z)=\beta(z)[\lambda f(\omega)+\gamma g(\omega, t)] \frac{1}{z-\omega \pm i 0}+c \delta(\omega-z),
$$


where $c$ is an arbitrary constant that can be settled equal to 1 $(c=1)$. Then, using Eq. (39) in (37), we obtain

$$
\begin{aligned}
& \left(\omega_{0}-z\right) \beta(z)+\beta(z) \int_{0}^{\infty} \frac{[\lambda f(\omega)+\gamma g(\omega, t)]^{2}}{z-\omega \pm i 0} d \omega \\
& \quad+\gamma g(z, t)+\lambda f(z)=0,
\end{aligned}
$$

so that

$$
\beta(z)=-\frac{\lambda f(z)+\gamma g(z, t)}{\omega_{0}-z+\int_{0}^{\infty} \frac{[\lambda f(\omega)+\gamma g(\omega, t)]^{2}}{z-\omega \pm i 0} d \omega} .
$$

Up to the first order in the coupling constants, the resonances of the compound system can be calculated as the poles of $\beta(z)$, i.e., by the zeros of the following function of $z$ :

$$
\xi_{ \pm}(z):=\omega_{0}-z+\int_{0}^{\infty} \frac{[\lambda f(\omega)+\gamma g(\omega, t)]^{2}}{z-\omega \pm i 0} d \omega .
$$

Observe that if $\gamma=0$, we have the exact expression giving the resonances of the system without the interaction $\gamma V_{1}(t)$. This expression is formally identical to the one obtained for the case of the standard Friedrichs model given in Eq. (10) and should therefore have the same properties, which are discussed in Ref. [22]. Zeros of Eq. (42) are the resonances of the combined system. Note that these zeros are functions of the coupling constants $\lambda$ and $\gamma$ as well as of the time.

Under certain conditions on Eq. (10), such as the analyticity of the square of the form factor $f(\omega)$ on an open set including the positive semiaxis $[0, \infty)$ and the existence of the Cauchy principal value in Eq. (10), one shows [22] the existence of a analytic continuation of $\eta_{+}(x)$ in Eq. (10) to the lower half-plane with a single zero. This zero is the resonance pole for the standard Friedrichs model and varies analytically with the coupling constant $\lambda$. In addition, the decay rate, which is twice the absolute value of the imaginary part of the resonance pole, is given in terms of the coupling constant by the expression $\Gamma(\lambda)=2 \pi \lambda^{2}\left|f\left(\omega_{0}\right)\right|^{2}+o\left(\lambda^{3}\right)$. This result has been obtained by making use of the analyticity of $\eta(z)$ and the implicit function theorem [22].

Therefore, if we assume that $f(\omega)$ and $g(\omega, t)$ are analytic functions with respect to $\omega$ in a domain including the positive semiaxis and the Cauchy principal value in Eq. (42) exists, then the same proof will show the following:

(i) There exists a resonance pole given by a zero of $\xi_{+}(z)$, which is an analytic function of the real form factors $\lambda$ and $\gamma$. If these form factors go to zero, then the resonance pole goes to the bound state of energy $\omega_{0}$.

(ii) The decay rate in terms of the form factors is given by the following expression:

$$
\Gamma(\lambda, \gamma, t)=2 \pi\left|\lambda f\left(\omega_{0}\right)+\gamma g\left(\omega_{0}, t\right)\right|^{2},
$$

where the terms proportional to the third power and higher in the coupling constants have been neglected.

While the nonvanishing value of the coupling $\lambda$ suffices for the appearance of resonances, the inclusion of a timedependent interaction (represented by a nonvanishing value of the coupling constant $\gamma$ ) can eventually suppress a resonant behavior. Naturally, we have assumed that both couplings are comparable magnitudes and that their absolute value is such that the obtained width $\Gamma$ is indeed much smaller than the real part of the energy of the state.

Equation (43) is a sort of generalization of the Fermi golden rule.

Note that both the localization of resonance poles and, consequently, the decay rate depend on the form of the potential. This means that the presence of an extra term may either enhance or diminish the tunneling probability. We have used a (general) time-dependent perturbation in order to compare our results with those obtained by Kälbermann $[20,21]$. In our first example in Sec. III, we show that the addition of a Dirac delta interaction to a finite square well with hard core at the origin enhances or diminishes the decay rate of the well resonances depending on the parameters of the added interaction. In our second example, using an interaction over the ordinary Friedrichs model, we observe that the variation of the decay rate depends solely on the shape of the interaction. In conclusion, the results obtained by Kälbermann are a consequence of the specific models used and they cannot be considered a general property of resonances under the action of interactions.

\section{CONCLUSIONS}

In this paper we have studied the coupling between resonances and the continuum. We have started by determining the resonant spectra of Friedrichs model and adding to it a coupling with the continuum, which was modeled by an extra time-dependent interaction. The modifications of the decay properties of the resonance were then shown to be a natural consequence of the added interaction. The solution was shown to be analytical in the coupling constants for a large class of interactions, which may enhance or reduce the decay rate. We have also studied the consequences, upon the resonancés decay properties, of adding a Diracdelta potential to a one-dimensional finite square well, and obtained similar results.

In our calculations, we have confronted the main conclusions of the work of Kälbermann, without restricting the analysis to a specific type of interactions.

In summary, it may be possible to change the decay rate of a resonance by exposure to external fields, as claimed by Kälbermann. However, the results are strongly dependent on the model used.

The problem now, from the physical point of view, is the realization of initially isolated resonances which should live long enough to feel the action of external fields.

\section{ACKNOWLEDGMENTS}

This work has been partially supported by the CONICET of Argentina (PIP 0740) and the Spanish Government Grants No. PR2011-0343, No. FPA2008- 04772-E, and No. MTM200910751. 
[1] G. Gamow, Z. Phys. 51, 204 (1928).

[2] T. Berggren, Nucl. Phys. A 109, 265 (1968).

[3] W. J. Romo, Nucl. Phys. A 116, 617 (1968).

[4] D. S. Delion, A. Insolia, and R. J. Liotta, Nucl. Phys. A 549, 407 (1992).

[5] A. Bohm and M. Gadella, Dirac Kets, Gamow Vectors and Gelfand Triplets, Lecture Notes in Physics Vol. 348 (Springer, Berlin, New York, 1988).

[6] O. Civitarese and M. Gadella, Phys. Rep. 396, 41 (2004).

[7] I. E. Antoniou and I. Prigogine, Physica A 192, 443 (1993).

[8] A. Bohm, I. Antoniou, and P. Kielanowski, J. Math. Phys. 36, 2593 (1995).

[9] I. Antoniou, Yu. Melnikov, Chaos Solitons Fractals 12, 2603 (2001).

[10] R. de la Madrid, A. Bohm, and M. Gadella, Fortschr. Phys. 50, 185 (2002).

[11] I. E. Antoniou, M. Gadella, E. Karpov, I. Prigogine, G. Pronko, Chaos Solitons Fractals 12, 2757 (2001).

[12] E. Hernández and A. Mondragón, Phys. Lett. B 326, 1 (1994).

[13] A. Mondragón, E. Hernández, and J. M. V. Arcos, Ann. Phys. (NY) 48, 503 (1991).
[14] R. G. Newton, Scattering Theory of Waves and Particles (Springer, New York, 1982).

[15] H. M. Nussenzweig, Causality and Dispersion Relations (Academic, New York, 1972).

[16] K. O. Friedrichs, Commun. Pure Appl. Math. 1, 361 (1948).

[17] M. Gadella, G. P. Pronko, Fortschr. Phys. 59, 795 (2011).

[18] O. Civitarese and M. Gadella, Int. J. Mod. Phys. E 15, 1273 (2006).

[19] O. Civitarese, M. Gadella, and G. P. Pronko, Int. J. Mod. Phys. E 16, 169 (2007).

[20] G. Kälbermann, Phys. Rev. C 77, 041601(R) (2008).

[21] G. Kälbermann, Phys. Rev. C 79, 024613 (2009).

[22] P. Exner, Open Quantum Systems and Feynmann Integrals (Reidel, Dordrecht, 1985).

[23] A. Bohm, M. Gadella, and B. G. Mainland, Am. J. Phys. 57, 1103 (1989).

[24] O. Civitarese, in Latin-American School of Physics-XL ELAF: Symmetries in Physics, AIP Conf. Proc. No. 1334 (AIP, New York, 2011), p. 54. 